\title{
Nonlinear Alfvén Waves in a Vlasov Plasma
}

Bell, T.F.

Published in:

Physics of Fluids

Link to article, DOI:

10.1063/1.1761115

Publication date:

1965

Document Version

Publisher's PDF, also known as Version of record

Link back to DTU Orbit

Citation (APA):

Bell, T. F. (1965). Nonlinear Alfvén Waves in a Vlasov Plasma. Physics of Fluids, 8(10), 1829-1839. https://doi.org/10.1063/1.1761115

\section{General rights}

Copyright and moral rights for the publications made accessible in the public portal are retained by the authors and/or other copyright owners and it is a condition of accessing publications that users recognise and abide by the legal requirements associated with these rights.

- Users may download and print one copy of any publication from the public portal for the purpose of private study or research.

- You may not further distribute the material or use it for any profit-making activity or commercial gain

- You may freely distribute the URL identifying the publication in the public portal

If you believe that this document breaches copyright please contact us providing details, and we will remove access to the work immediately and investigate your claim. 


\title{
Nonlinear Alfvén Waves in a Vlasov Plasma
}

\author{
T. F. BELI \\ Ionosphere Laboratory, Technical University of Denmark, Lyngby, Denmark
}

(Received 26 April 1965)

\begin{abstract}
Stationary solutions to the nonlinear Vlasov-Boltzmann equations are considered which represent one-dimensional electromagnetic waves in a hot magnetoplasma. These solutions appear in arbitrary reference frames as circularly polarized, sinusoidal waves of unlimited amplitude, i.e., as nonlinear Alfvén waves. Solutions are found implicitly by deriving a set of integral dispersion relations which link the wave characteristics with the particle distribution functions. A physical discussion is given of the way in which the Alfven waves can trap particles, and it is shown that the presence of "cyclotron-trapped" particles introduces a boundary condition which no set of isotropic distribution functions can satisfy. An application of these solutions to theories of very low frequency emissions is briefly discussed.
\end{abstract}

\section{INTRODUCTION}

$\mathbf{I}^{\mathrm{N}}$ $\mathrm{N}$ the present paper we wish to show how solutions to the nonlinear Vlasov-Boltzmann equations can be constructed which represent sinusoidal, circularly polarized plane electromagnetic waves propagating parallel to the direction of a constant magnetic field in a hot plasma. This plasma is infinite and free from collisions and consists entirely of electrons and various kinds of ions. The nonlinear waves can be thought of as Alfvén or whistler mode ${ }^{1}$ waves of arbitrary amplitude since in the limit of the linear theory they correspond to the characteristic modes of propagation known variously as the Alfvén or whistler modes.

We limit ourselves to finding solutions which appear stationary in a particular frame of reference, i.e., we assume that there exists a rest frame for the wave. In this rest frame we construct "solutions" to the nonlinear Vlasov-Boltzmann equations in the sense that we derive a set of "dispersion" relations, involving the particle distribution functions, which implicitly define those sets of particle distribution functions which will satisfy the nonlinear VlasovBoltzmann equations. These integral relations are given in a form which does not involve any spatial variables and in which, given a particular set of distribution functions, all integrals may be directly evaluated. The generality of the wave types we consider is restricted; the dispersion relations apply only to sinusoidal Alfvén waves. Nevertheless, these nonlinear solutions are important in at least two respects:

(1) They allow an investigation of the effects of trapped particles upon an electromagnetic wave of arbitrary amplitude in a Vlasov plasma.

(2) They represent an extension into the non-

1 T. H. Stix, The Theory of Plasma Waves (McGraw-Hill Book Company, Inc., New York, 1962), Chap. 9. linear regime of a mode of electromagnetic wave propagation which in the linear theory is a characteristic mode of propagation, i.e., the Alfvén or whistler mode.

Nonlinear Alfvén waves in a cold plasma have been discussed previously by a number of workers. ${ }^{2}$ However, these cold plasma nonlinear solutions cannot take proper account of particle trapping, and in addition, their usefulness in extending the linear theory of the Vlasov plasma is limited. It must be remarked, however, that these cold plasma solutions have the virtue of simplicity and are also applicable to a wide variety of wave forms; these are two advantages over the solution given here.

A method of constructing stationary nonlinear solutions to the Vlasov-Boltzmann equations has been outlined by Bernstein. ${ }^{3}$ This method involves first determining the constants of the motion of the individual particle trajectories under the influence of the static fields and then determining those distribution functions which, when expressed in terms of the constants of the motions, satisfy the Maxwell equations, as well as appropriate subsidiary conditions. The main point to this development is that the distribution functions, when expressed in terms of the particle constants of the motion, automatically satisfy the Boltzmann equation in the wave rest frame. $^{3,4}$ Once a solution is obtained in the rest frame then the solution in any other frame may be obtained by means of a simple Galilean or Lorentz transformation. This method has been used by a

${ }^{2}$ See, for example, V. C. A. Ferrarro, Proc. Roy. Soc. (London) A233, 310 (1955), or D. C. Montgomery, Phys. Fluids 2, 585 (1959).

${ }^{3}$ I. B. Bernstein, in Radiation and Waves in Plasmas, edited by M. Mitchner (Stanford University Press, Stanford, California, 1961), p. 19.

${ }^{4} \mathrm{~S}$. Chandrasekhar, Plasma Physics, notes compiled by S. Trehan (The University of Chicago Press, Chicago, Illinois, 1962). 
number of workers to construct stationary nonlinear solutions which represent one-dimensional plasma oscillations. $^{5}$

In the present paper we consider a one-dimensional situation involving electromagnetic waves, a case which is somewhat more complex than that of the plasma oscillations. In Sec. II the general dispersion relations for the particle distribution functions are developed, and in Sec. III the physical nature of the particle trapping by the Alfvén wave is discussed. Section IV is devoted to a phase analysis of the particle trajectories and to the statement of the boundary conditions which the presence of gyroresonant trapped particles impresses upon the distribution functions. A brief discussion of the results follows in Sec. V.

\section{ANALYSIS}

Consider a plane electromagnetic wave which is propagating parallel to a static magnetic field in an infinite plasma consisting of electrons and various kinds of ions. Let the static magnetic field $\mathbf{B}_{0}$ point along the positive $z$ axis in a right-handed Cartesian coordinate system, and let the field components of the wave be functions of $z$ alone. Given now that a wave rest frame exists and that this frame translates in the laboratory system with a velocity less than that of light, the only nonzero components of the fields in this frame will be

$$
\begin{array}{ll}
E_{z}=E_{z}(z), & B_{z}=B_{0}, \\
B_{x}=B_{x}(z), & B_{y}=B_{y}(z),
\end{array}
$$

where $B_{x}$ is the component of $\mathbf{B}$ along the $x$ axis in the rest frame, etc. In general the presence of the longitudinal electric field will couple the electromagnetic wave to the longitudinal plasma oscillations, and this is a complication which it is wished to avoid. Therefore, we take $E_{\star}=0$, a restriction which means that in the laboratory frame the wave will appear purely transverse.

In the rest frame the distribution function for the $i$ th type of particle $f^{(i)}$ must satisfy the stationary collisionless Boltzmann equation.

$$
(\nabla \cdot \nabla) f^{(i)}+\eta_{i}(\nabla \times \mathrm{B}) \cdot \nabla_{\mathrm{v}} f^{(i)}=0,
$$

where $B$ is the total magnetic field in the rest frame, $\eta_{i}$ is the ratio of charge to mass for $i$ th type of particle, and $\nabla, \nabla_{\nabla}$ are the Laplacian operators in coordinate and velocity space, respectively.

The Maxwell set can be written ${ }^{3}$

- I. B. Bernstein, J. M. Greene, and M. D. Kruskal, Phys. Rev. 108, 546 (1957); also, see the paper by D. Bohm and E. P. Gross, Phys. Rev. 75, 1851 (1949).

$$
\begin{aligned}
\frac{\partial B_{x}}{\partial z} & =\mu_{0} \sum_{i} q_{i} \int v_{y} f^{(i)} d \mathbf{v}, \\
\frac{\partial B_{y}}{\partial z} & =-\mu_{0} \sum_{i} q_{i} \int v_{x} f^{(i)} d \mathbf{v}, \\
j_{z} & =0=\sum_{i} q_{i} \int v_{z} f^{(i)} d \mathbf{v}, \\
\rho & =0=\sum_{i} q_{i} \int f^{(i)} d \mathbf{v},
\end{aligned}
$$

where $j_{z}$ is the $z$ component of the total current density, $\rho$ is the total charge density, $q_{i}$ is the charge of the $i$ th type of particle, $d \mathbf{v}=d v_{x} d v_{y} d v_{z}$, and the integrals are to be taken over the whole of velocity space.

The problem is now to determine those sets of distribution functions $f^{(i)}$ which will satisfy Eqs. (2.1)-(2.5), along with the subsidiary conditions, introduced for physical reasons, that there be no particles of negative number density, that $f^{(i)}$ be normalizable, and that the momentum and energy density of the particles be finite, i.e.,

$$
\begin{gathered}
f^{(i)} \geq 0, \\
\int f^{(i)}\left|v_{i}\right|^{m} d \mathrm{v} \leq M,
\end{gathered}
$$

where $j=x, y, z, \quad m=0,1,2$, and $M$ is some finite number. It can be demonstrated that any function (sufficiently smooth) which depends only upon the constants of the motion of the particle trajectories in the rest frame will satisfy Eq. (2.1). Our procedure is to determine these constants of the motion, express the $f^{(i)}$ in terms of the constants, and then to establish under what conditions these $f^{(i)}$ will satisfy the Maxwell set, as well as Eqs. (2.6) and (2.7).

We determine the appropriate constants of the particle motion by solving the equations of motion of a particle of charge to mass ratio $\eta_{i}$ under the influence of the static fields.

These equations are given by the Lorentz law:

$$
\dot{\mathrm{v}}=\eta_{i}[\mathbf{v} \times \mathbf{B}] \text {, }
$$

where the dot indicates a total time derivative. We restrict our attention to waves which appear in the laboratory frame as sinusoidally varying, circularly polarized waves. In this case in the rest frame the transverse magnetic field $B_{w}$ will have the form $\mathbf{B}_{w}(z)=B_{w}\left[\mathbf{e}_{x} \cos k\left(z-z_{0}\right) \pm \mathbf{e}_{u} \sin k\left(z-z_{0}\right)\right]$,

where $B_{w}$ is the amplitude of the rotating variable magnetic field, $k$ is the wavenumber of the static field, $\mathbf{e}_{x}, \mathbf{e}_{y}$ are unit vectors in the $x$ and $y$ direction, 
and $z_{0}$ is some constant. For explicitness we chose the positive sign in Eq. (2.9), but the choice of the minus sign would represent no difficulty in the development to follow. [Note that the substitution $k^{\prime}=-k$ is equivalent to a change of sign in Eq. (2.9).]

The derivation of the relevant constants of the motion from Eq. (2.8) is best performed in a coordinate system described by the triple $\left(v_{z}, v_{\perp}, \phi\right)$. Here $v_{z}$ is the particle velocity in the $z$ direction, $v_{\perp}$ is the magnitude of the particle velocity perpendicular to the $z$ axis, and $\phi$ is the angle between $\mathbf{B}_{w}$ and $\boldsymbol{\nabla}_{\perp}$ measured in a positive sense from $\mathbf{B}_{w}$ to $\nabla_{\perp}$ so that

$$
\begin{aligned}
\mathbf{B}_{w} \times \nabla_{\perp} & =\mathbf{e}_{z}\left|\mathbf{B}_{w}\right|\left|\mathbf{v}_{\perp}\right| \sin \phi, \\
\mathbf{B}_{w} \cdot \mathbf{v}_{\perp} & =\left|\mathbf{B}_{w}\right|\left|\mathbf{v}_{\perp}\right| \cos \phi
\end{aligned}
$$

(see Fig. 1). In this coordinate system the equations of motion (2.8) have the following representation:

$$
\begin{aligned}
\dot{v}_{z} & =-\eta_{i} B_{w} v_{\perp} \sin \phi, \\
\dot{v}_{\perp} & =\eta_{i} B_{w} v_{z} \sin \phi, \\
\dot{\phi} & =-k v_{z}-\eta_{i}\left[B_{0}-B_{w}\left(v_{z} / v_{\perp}\right) \cos \phi\right] .
\end{aligned}
$$

Two constants of the motion can be obtained from the above set of equations:

$$
\begin{aligned}
v_{z}^{2}+v_{\perp}^{2} & =E^{(i)}, \\
v_{\perp}^{2}+2 V_{c}^{(i)} v_{z}+2 V_{w}^{(i)} v_{\perp} \cos \phi & =F^{(i)},
\end{aligned}
$$

where

$$
V_{c}^{(i)}=-\left(\eta_{i} B_{0} / k\right) \text { and } V_{w}^{(i)}=-\left(\eta_{i} B_{w} / k\right) .
$$

[From this point on, the superscript $(i)$ will be suppressed with respect to $E, F, V_{c}$ and $\left.V_{w}.\right]$ Equation (2.11) is just an expression of the conservation of kinetic energy in the rest frame; (2.12) has the characteristics of angular momentum conservation. The deduction of these two constants is sufficient to enable us to find $v_{z}(t), v_{\perp}(t)$, or $\phi(t)$.

It is wished to express the velocity integrals in Eqs. (2.2)-(2.5) in terms of these constants of the motion. The third variable of integration will be somewhat arbitrary since we have only two constants of the motion. Choose the third variable of integration as the angle $\phi$. The coordinate transformation $\left(v_{x}, v_{y}, v_{z}\right) \rightarrow(E, F, \phi)$ relates the differential volume elements in the two spaces through the Jacobian $J$ of the transformation

$$
d E d F d \phi=J d v_{x} d v_{y} d v_{s},
$$

where

$$
J=\partial(E, F, \phi) / \partial\left(v_{x}, v_{y}, v_{z}\right) .
$$

FIG. 1. Display of normal and Cartesian coordinates for an arbitrary particle. The position vector of the particle in the $(x, y)$ plane has the value $I_{\perp}$. The angle $\psi$ is defined by $\psi=k\left(z-z_{0}\right)$.

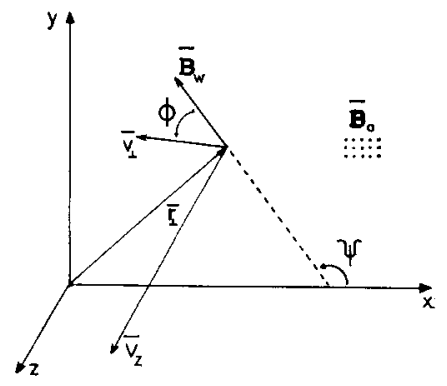

(As in the case of the quantities $E$ and $F$, it should be borme in mind in what follows that the quantity $J$ will in general be different for each particle species.)

The quantities $v_{x}$ and $v_{y}$ may be expressed in terms of $v_{\perp}$ and $\phi$ through the relations

$$
\begin{aligned}
& v_{x}=v_{\perp} \quad \cos \left(\phi+k z-k z_{0}\right), \\
& v_{y}=v_{\perp} \quad \sin \quad\left(\phi+k z-k z_{0}\right),
\end{aligned}
$$

and the Jacobian above has the explicit value

$$
J=4(\dot{\phi} / k),
$$

where $\dot{\phi}$ is given as in Eqs. (2.10). Formally, a typical integral in Eq. (2.2), for instance, will now appear as

$I_{i}=\int d E \int d F \int v_{y}(E, F, \phi) f^{(i)}(E, F) \frac{d \phi}{J}$.

There are two immediate difficulties with Eq. (2.16) which must be dealt with. First, the transformation leading to Eq. (2.13) is not one-to-one, and consequently, in order to properly preserve phase space volume, Eq. (2.13) must be interpreted as applying separately to each real branch of $\dot{\phi}=\dot{\phi}(E, F, \phi)$. It can be shown from Eq. (2.11), Eq. (2.12), and the last of Eqs. (2.10) that there may be as many as four real branches of $\dot{\phi}$. In these cases the integrals involving $J$, such as Eq. (2.16), must be interpreted as being taken over all the real branches of $\dot{\phi}$ in order to preserve phase space volume. For our purposes it is convenient to perform formally all integrations over $\phi$ which appear in expressions such as Eq. (2.16) and in the future we indicate symbolically, by the subscript $s$ below the integrals, the particular branch of $\dot{\phi}$ along which the integration is to be performed.

The second point of difficulty with Eq. (2.16) rests with the distribution function $f^{(i)}$. Each real branch of $\phi$ will correspond to a different region of the $\left(v_{x}, v_{y}, v_{z}\right)$ space [as can be seen by the last of Eqs. (2.10)], and the value of $f^{(i)}$ need not be the same in each of these regions. This fact we indicate 
by the subscript $s$ attached to $f^{(i)}$. Equation (2.16) is now properly written

$$
I_{i}=\sum_{s} \int d E \int d F \int_{a} v_{v} f_{a}^{(i)} \frac{d \phi}{J_{s}} .
$$

The last point we make with regard to these integrals is that, by convention, we interpret all integrals as being taken in a positive sense and consider the Jacobian as always positive.

With the above considerations in mind, we can use Eqs. (2.9), (2.13), and (2.14) in order to transform Eqs. (2.2) and (2.3) into the following two equations which do not depend explicitly upon any space variables:

$$
\begin{aligned}
\frac{-k B_{w}}{\mu_{0}} & =\sum_{i, s} \int d E \int d F \int f_{s}^{(i)} v_{\perp} \cos \phi \frac{d \phi}{J_{s}}, \\
0 & =\sum_{i, s} \int d E \int d F \int f_{s}^{(i)} v_{\perp} \sin \phi \frac{d \phi}{J_{s}} .
\end{aligned}
$$

It is possible to show that Eq. (2.19) is always satisfied no matter what form the distribution functions possess. To see this, note that the integral over $\phi$ may be performed first, with $E$ and $F$ fixed. But from Eq. (2.12) it can be seen that the curves describing the particle motion for fixed $E$ and $F$ are symmetric about $\phi=0$. Thus on these curves, $v_{\perp}(\phi)=v_{\perp}(-\phi), v_{z}(\phi)=v_{z}(-\phi)$, and consequently, any branch of $\dot{\phi}$ will be symmetric about $\phi=0$. But $v_{\perp} \sin \phi$ is odd about $\phi=0$. Thus,

$$
\int_{\perp} v_{\perp} \sin \phi \frac{d \phi}{J_{s}}=0 \quad \text { for all } s
$$

and Eq. (2.19) is satisfied no matter what form the distribution functions possess.

Equations (2.4) and (2.5) may be transformed in a manner similar to Eq. (2.10), and if, in addition, we formally carry out the integrals in $\phi$, the entire set can be written

$$
\begin{aligned}
A & =\sum_{i, s} q_{i} \iint G_{s}^{(i)} f_{s}^{(i)} d E d F, \\
0 & =\sum_{i, s} q_{i} \iint H_{s}^{(i)} f_{s}^{(i)} d E d F, \\
0 & =\sum_{i, s} q_{i} \iint T_{s}^{(i)} f_{s}^{(i)} d E d F,
\end{aligned}
$$

where

$$
\begin{aligned}
A & =-k B_{w} / \mu_{0}, \quad G_{s}^{(i)}=\int_{s} v_{\perp} \cos \phi d T, \\
H_{a}^{(i)} & =\int_{s} v_{z} d T, \quad T_{s}^{(i)}=\int_{s} d T, d T=d \phi / J_{s} .
\end{aligned}
$$


with constant velocity $v_{z}^{\prime}$ in the $z$ direction while rotating about a particular field line of $\mathbf{B}_{\mathbf{0}}$ with an angular velocity $\omega_{0}$ equal to the electron cyclotron frequency, $\omega_{\mathrm{c}}=e B_{0} / m$. The sense of this rotation would be such that the angular momentum vector associated with the electron rotation would be directed along the $z$ axis. The pitch of the helix $\psi$ would be given by the relation $\psi=\tan ^{-1}\left(v_{\perp}^{\prime} / v_{z}^{\prime}\right)$. In the combined fields of the Alfvén wave and the constant magnetic field, it is also possible for the electron to move in a helical path of constant pitch about a particular field line of $\mathbf{B}_{\mathbf{0}}$. This can be seen in the following way. First assume that the electron is observed at some instant of time at which it has the (nonzero) velocity components $v_{z}^{\prime}$ parallel to $\mathbf{B}_{0}$ and $v_{1}^{\prime}$ perpendicular to $\mathbf{B}_{0}$. In this case there are two forces on the electron in a plane perpendicular to $B_{0}$. One of these is due to the action of the constant magnetic field $\mathbf{B}_{0}$ and has the form

$$
\mathbf{F}_{1}=-e\left(\mathbf{v}_{\perp}^{\prime} \times \mathbf{B}_{0}\right),
$$

while the second is due to the action of the wave magnetic field $\mathbf{B}_{w}$ and has the form

$$
\mathbf{F}_{2}=-e\left(\mathbf{v}_{z}^{\prime} \times \mathbf{B}_{w}\right) \text {. }
$$

In addition there is a third force which acts along the $z$ direction and which has the form

$$
F_{z}=-e\left(\mathbf{v}_{\perp}^{\prime} \times \mathbf{B}_{w}\right) \text {. }
$$

Uniform helical motion of the electron is possible only if the magnitudes of the velocity components remain constant. A necessary condition for this is $F_{z}=0$. Note that this implies that $\mathbf{v}_{\perp}^{\prime}$ and $\mathbf{B}_{w}$ are either parallel or antiparallel, and consequently, $\mathbf{F}_{2}$ must be perpendicular to $\mathbf{v}_{1}^{\prime}$. But $F_{1}$ is always perpendicular to $\nabla_{\perp}^{\prime}$, and when $F_{2}$ also is, we may find the instantaneous angular velocity of the electron $\omega$ about $\mathbf{B}_{0}$ by equating the centripetal and centrifugal forces:

$$
m \omega v_{\perp}^{\prime}=e\left(v_{\perp}^{\prime} B_{0} \pm v_{z}^{\prime} B_{w}\right),
$$

where the \pm arises from the fact that $\nabla_{\perp}^{\prime}$ and $\mathbf{B}_{w}$ may be either parallel or antiparallel. Furthermore, as a result of its velocity along the $z$ axis, the electron will see the transverse magnetic field $\mathbf{B}_{v}$ appear to rotate in the $x, y$ plane at the angular Doppler frequency

$$
\omega_{\mathrm{d}}=k v_{z}^{\prime} .
$$

The sense of this rotation will be the same as that of the electron's rotation about $\mathbf{B}_{0}$ as long as both $\omega$ and $\omega_{\mathrm{d}}$ have the same sign. Given that $F_{z}$ is initially zero, it is clear that $F_{z}$ will remain zero so long as $\omega=\omega_{\mathrm{d}}$ and the phase between $\mathbf{B}_{w}$ and $\nabla_{\perp}$ remains constant. But as long as $F_{z}$ is zero, $v_{z}$ will not change from its initial value $v_{z}^{\prime}$ and $\omega_{\mathrm{d}}$ will remain constant. In addition, as long as $F_{z}$ is zero, $F_{2}$ will remain perpendicular to $v_{\perp}$, there will be no forces parallel to $v_{\perp}$, the magnitude of $v_{\perp}$ will remain at its initial value $v_{\perp}^{\prime}$, and $\omega$ will remain constant. But the condition $\omega=\omega_{\mathrm{d}}$ ensures that $F_{z}$ will remain zero, and thus, the entire configuration will have no tendency to change with time. The conditions for uniform helical motion are then just that at any time:

$$
\omega=\omega_{\mathrm{d}}, \quad \mathbf{v}_{\perp} \times \mathbf{B}_{w}=0,
$$

or equivalently

$$
e / m\left[B_{0} \pm\left(v_{z} / v_{\perp}\right) B_{w}\right]=k v_{z}, \quad \sin \phi=0,
$$

where $\phi$ is the angle between $\mathbf{v}_{\perp}$ and $\mathbf{B}_{w}$, as defined in Sec. II. It will be noted that the above relations are equivalent to those that can be derived from Eqs. (2.10) by requiring that $\dot{v}_{\perp}=\dot{v}_{z}=\dot{\phi}=0$. Thus uniform helical motion is the motion determined by the singular points of (2.10) and is the fundamental "average" motion of any particle trapped by the Alfvén wave. The deviations from the uniform helical motion can be expressed in terms of the angle $\phi$ between $\mathbf{v}_{\perp}$ and $\mathbf{B}_{w}$. The trapped particles are marked by the fact that, for their motion, the variation in $\phi$ is bounded, $|\phi|<\pi$, and the transverse velocity vectors of these particles tend to be bunched with respect to phase about $\mathbf{B}_{0}$. Thus, we have the important point that in any plane perpendicular to $\mathbf{B}_{0}$ the velocity vectors of the trapped particles will be in approximately the same direction, and these particles will act as a coherent current source.

It may be noted that for fixed $B_{0}$ and $B_{w}$, Eq. (3.6) appears to be satisfied by an infinite range of values of $v_{z}$ and $v_{\perp}$; however, it must be borne in mind that the initial kinetic energy of any single particle is a definite quantity and $v_{\perp}$ and $v_{z}$ must satisfy the relationship $v_{\perp}^{2}+v_{z}^{2}=$ const. Taking this fact into account, it can be shown that there are at most four ways to satisfy Eq. (3.6) for a given value of kinetic energy. It is instructive to list briefly the possibilities according to their qualitative features.

(i) The quantity $\alpha \equiv\left|v_{z} B_{w} / v_{\perp} B_{0}\right|$ is large, and $v_{z}$ is negative. Here the motion is controlled mainly by $\mathbf{B}_{w}$, which actually reverses the sense of rotation of the electron about $\mathbf{B}_{\mathbf{0}}$.

(ii) $\alpha$ is large, and $v_{z}$ is large and positive. Here $B_{w}$ controls again, causing a large increase in $\omega$ above $\omega_{\mathrm{e}}$. 
(iii) $\alpha$ is small, and $v_{z}=\omega_{\mathrm{c}} / k+\epsilon$, where $\epsilon$ has two values, one small and negative, and one small and positive. Here the motion is controlled mainly by the field $\mathbf{B}_{0}$, and the particle rotates about $\mathbf{B}_{0}$ at approximately its unperturbed cyclotron frequency $\omega_{\mathrm{c}}$.

Since (iii) requires that $v_{z}$ have a certain minimum value, the conditions there cannot be satisfied for all values of kinetic energy. However, given that some one value of kinetic energy is high enough to satisfy (iii), any higher value of kinetic energy can also satisfy (iii), and thus this trapping region can affect particles over a wide range of energy.

It is instructive to note that in the limit of the linear theory as $B_{w} \rightarrow 0$, the two values of $v_{z}$ in (iii) tend to one, $v_{z} \rightarrow \omega_{0} / k$. But this is just the condition in the linear theory (applied to the rest frame) that the particle be in cyclotron resonance with the Alfven wave. In view of this correspondence with the linear theory, we choose to label the trapping characterized by the conditions of (iii) as cyclotron trapping. In Sec. IV it is brought out that the presence of cyclotron-trapped particles introduces certain boundary conditions which restrict the form of the allowable particle distribution functions.

\section{INTERPRETATION OF INTEGRALS}

In this section we wish to define the limits of the integrals in Eqs. (2.20)-(2.22) and to determine the domains of the distribution functions $f_{s}^{(i)}$, as well as any boundary conditions on $f_{s}^{(i)}$ which might be appropriate. In order to achieve these ends, we need to know the limits of the $(E, F)$ parameter space, the distribution of the branches of $\dot{\phi}$, and the location of the singular points of Eqs. (2.10). This information can be obtained by means of a phase plane study of the particle motions, which we outline briefly below.

\section{A. Phase Plane Study}

Our phase plane study is in essence a study of that family of integral curves which is defined by the intersection of Eqs. (2.11) and (2.12). Since Eq. (2.11) is just the equation of a sphere of radius $E^{\frac{2}{2}}$ in a Cartesian velocity space, the integral curves will lie upon the surface of this sphere and can be represented conveniently in spherical coordinates in velocity space. In order to transform into the spherical coordinate system, we introduce the colatitudinal coordinate $\theta$ defined so that

$$
\begin{aligned}
& v_{z}=E^{\frac{1}{2}} \cos \theta, \quad(0 \leq \theta \leq \pi) \\
& v_{\perp}=E^{\frac{1}{2}} \sin \theta,
\end{aligned}
$$

and we let the angle $\phi$ go over into a longitudinal angle on the sphere. We also introduce the dimensionless parameters

$$
\begin{aligned}
r & =B_{w} / B_{0}, \\
\delta & =\left|\eta_{i}\right|\left(\hat{\eta}_{i}\right)^{-1}= \pm 1, \\
\lambda & =E^{\frac{1}{2}}\left|V_{\mathrm{o}}\right|, \\
\sigma & =F V_{\mathrm{o}}^{-2},
\end{aligned}
$$

and the dimensionless "time," $d \xi=B_{0} \eta_{i} d t$.

In terms of these new quantities, Eqs. (2.10) become

$\dot{\theta}=-\delta r \sin \phi$,

$\dot{\phi}=1-\lambda \cos \theta-\delta r \cot \theta \cos \phi$,

(the dot denotes $d / d \xi$ ) with the constant of the motion

$\lambda\left[\lambda \sin ^{2} \theta+2 \cos \theta+2 \delta r \sin \theta \cos \phi\right]=\sigma$.

Given $r$ and $\delta$ fixed, Eq. (4.2) represents a twoparameter $(\sigma, \lambda)$ family of curves. Given also $\sigma$, and $\lambda$ fixed, then Eq. (4.2) describes all the possible real motions available to any particle characterized by the given values of $\sigma$ and $\lambda$. It is possible to show that the real particle motions governed by Eq. (4.1) and satisfying Eq. (4.2) will correspond to curves which are either closed on the surface of the velocity space sphere or else consist of a single point on this surface. Let us define each of these closed curves (or single points) as a trajectory. Since Eq. (4.1) obeys a Lipschitz condition, ${ }^{7}$ these trajectories will be unique and each will correspond to an entire physical motion of a particle. ${ }^{8}$ Through geometrical arguments, it can be demonstrated that there will be at most two real trajectories for any given $\sigma$ and $\lambda$, and thus at most two entire physical motions in the velocity space.

As discussed in Sec. III, the motions characteristic of trapped particles will be those for which $\phi$ is bounded, $|\phi|<\pi$. In this regard, the phase space $(\theta, \phi)$ can be divided into those regions which contain trapped particle trajectories and those which do not. The singular points of Eqs. (4.1) will define some of the limits of these trapping regions. Inspection of Eqs. (4.1) shows that the singular points are defined by the relations

$$
\phi_{s}=n \pi, \quad|n|=0,1,2, \ldots,
$$

${ }^{7}$ E. A. Coddington and N. Levinson, Theory of Ordinary Differential Equations (McGraw-Hill Book Company, Inc., New York, 1955).

${ }^{8} \mathrm{~N}$. Minorsky, Nonlinear Oscillations (D. Van Nostrand Company, Inc., Princeton, New Jersey, 1962). 


$$
\begin{gathered}
0=1-\lambda \quad \cos \theta_{s}-\delta r(-1)^{n} \cot \theta_{s}, \\
0 \leq \theta_{s} \leq \pi \quad \text { (by definition). }
\end{gathered}
$$

As a result of Eq. (4.5), the real values of $\theta_{\text {a }}$ which satisfy Eq. (4.4) will be unambiguously defined if $\cos \theta_{3}$ is known. The real values of $\cos \theta_{0}$ which satisfy (4.4) also satisfy the polynomial relation divided from (4.4)

$$
P(X)=\left(\lambda^{2}-X^{2}\right)(1-X)^{2}-r^{2} X^{2}=0,
$$

in which $X=\lambda \cos \theta_{a}$.

The following properties of $P(X)$ may be readily shown assuming that $\lambda$ and $r$ are real and nonzero:

(A) $P(X)$ possesses a unique real negative root $X_{1}$ and at least one positive real $\operatorname{root} X_{2}$ for all $\lambda>0$.

(B) Define $\lambda_{c}=\left[1+r^{3}\right]^{\frac{3}{2}}$. For $\lambda<\lambda_{c}, P(X)$ possesses only two real roots $X_{1}$ and $X_{2}$. For $\lambda \geq \lambda_{c}$, $P(X)$ possesses four real roots, which we order $X_{1}<0<X_{2}<X_{3} \leq X_{4}$. The condition $X_{3}=X_{4}$ applies only when $\lambda=\lambda_{c}$.

(C) Each distinct real root of $P(X)$ defines the $\theta$ coordinate of a distinct singular point.

Since Eqs. (4.1) admit an integral of the motion, Eq. (4.2), the singular points defined by Eqs. (4.1), are either centers or saddle points or points which represent the confluence of a center and saddle point. ${ }^{8}$ For present purposes we refer to the centers as stable points and to the saddle points as unstable points.

Let us define the values of $\theta_{s}$ which correspond to the real roots $X_{i}$ of $P(X)$ as $\theta_{i}$, i.e.,

$$
\theta_{i}=\cos ^{-1}\left(X_{i} \lambda^{-1}\right),
$$

where the $X_{i}$ are given as in (B) above. We denote by the symbol $\phi_{i}$ the principal value of $\phi_{s}$ appropriate to the singular point whose $\theta_{8}$ value is $\theta_{i}$. Analysis of Eqs. (4.1) through the variational method $^{8}$ of Poincare shows that the singular points $\left(\theta_{i}, \phi_{i}\right)$ have the following properties as functions of $\lambda$ :

$$
\lambda<\lambda_{\mathrm{c}} \text {. }
$$

Here there are two singular points defined by Eqs. (4.3)-(4.5), both of which are stable. The values of $\theta_{i}$ for these points are defined through Eq. (4.7) and (B). The values of $\phi_{i}$ for these two singular points are $\phi_{1}=\frac{1}{2} \pi(1+\delta), \phi_{2}=\frac{1}{2} \pi(1-\delta)$.

$$
\lambda=\lambda_{\mathrm{s}} \text {. }
$$

There are two stable singular points for which $\theta_{i}$ is defined by Eq. (4.7), the $X_{i}$ referring to the negative root and the smallest positive root of $P(X)$ [i.e., $X_{1}$ and $X_{2}$ in (B) above]. There is also a singular point, $\left(\theta_{3}, \phi_{3}\right)$ corresponding to the double root $X_{3}=X_{4}$, which represents the confluence of a center and a saddle point. This higher order singular point does not represent a stable particle motion. In these three cases, $\phi_{1,2}$ are as in (a) above, while $\phi_{3}=\phi_{1}$.

$$
\lambda>\lambda_{c} \text {. }
$$

There are three stable singular points for which $\theta_{i}$ is defined by Eq. (4.7), the $X_{i}$ referring to the negative root and the smallest and largest positive roots of $P(X)$ [i.e., $X_{1}, X_{2}$ and $X_{4}$ in (B) above]. There is also one unstable singular point $\left(\theta_{3}, \phi_{3}\right)$ corresponding to $X_{3}$. The associated points $\phi_{i}$ for $i=1,2$ are as in (a) above; also $\phi_{3}=\phi_{4}=\phi_{1}$.

The stable singular points will be enclosed by trajectories for which the variation of $\phi$ is bounded and these curves fill out certain portions of the $(\theta, \phi)$ phase plane, portions which we refer to as the trapping regions. Each of the trapping regions is bounded by a separatrix which is the limiting curve separating the trapped orbits from the untrapped. (A separatrix is not itself a trapped orbit, and it may not connect the unstable singular points.) For a fixed value of $\lambda$ the separatrix for each of the possible trapping regions may be defined in terms of a value of $\sigma$ through Eq. (4.2).

Let us denote the value of $\sigma$ at any of the singular points $\left(\theta_{i}, \phi_{i}\right)$ as $\sigma_{i}=\sigma_{i}(\lambda)$. Let us also denote the region of trapping in the $(\theta, \phi)$ plane about the stable singular points $(i=1,2,4)$ as $R_{i}$. Finally, let us denote as $\lambda_{d}$ the value of $\lambda$ defined implicitly through the relations $\sigma_{3}(\lambda)=2 \lambda ; \lambda>\lambda_{0}$. Given these definitions, the following statements may be made with regard to the limits of the regions $R_{i}$ :

(1) Region $R_{1}$ is bounded by the separatrix $\sigma(\theta, \phi)=-2 \lambda$, where $\sigma(\theta, \phi)$ represents the left-hand side of $\mathrm{Eq}$. (4.2). The trapping region consists of all orbits characterized by a value of $\sigma$ in the interval $\sigma_{1} \leq \sigma<-2 \lambda$. This is true for all $\lambda$.

(2) Region $R_{2}$ is bounded by the separatrix $\sigma(\theta, \phi)=2 \lambda$, for values of $\lambda$ in the interval $0<$ $\lambda\left\langle\lambda_{\mathrm{d}}\right.$, while for $\left.\lambda\right\rangle \lambda_{\mathrm{d}}$ the region is bounded by the separatrix $\sigma(\theta, \phi)=\sigma_{3}$. The trapping region consists of all orbits characterized by a value of $\sigma$ in the interval:

$$
\begin{array}{ll}
2 \lambda<\sigma \leq \sigma_{2} ; & 0 \leq \lambda \leq \lambda_{d} \\
\sigma_{3}<\sigma<\sigma_{2} ; & \lambda>\lambda_{d} .
\end{array}
$$

(3) Region $R_{4}$ exists only for $\lambda>\lambda_{0}$. For values of $\lambda$ in the interval $\lambda_{\mathrm{c}}<\lambda \leq \lambda_{\mathrm{d}}$, this region is bounded by the separatrix $\sigma(\theta, \phi)=\sigma_{3}$, while for 


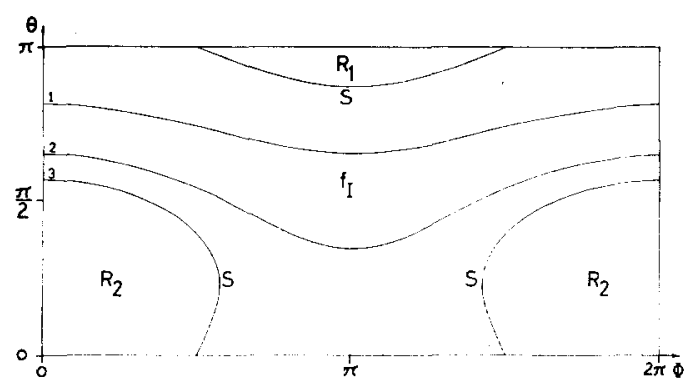

Fig. 2. Sketch of typical phase plane trajectories for $\lambda<\lambda_{c}$. The particle density on any of these trajectories for a given species can be described by a single distribution function $f_{\mathrm{I}}$.

$\lambda>\lambda_{\mathrm{d}}$, the region is bounded by the separatrix $\sigma(\theta, \phi)=2 \lambda$. The trapping region consists of all orbits characterized by a value of $\sigma$ in the interval:

$$
\begin{array}{ll}
\sigma_{4} \leq \sigma<\sigma_{3} ; & \lambda_{\mathrm{c}}<\lambda \leq \lambda_{\mathrm{d}}, \\
\sigma_{4} \leq \sigma<2 \lambda ; & \lambda>\lambda_{\mathrm{d}} .
\end{array}
$$

The above statements comprise the essential points of the phase plane study and can be made to apply explicitly to any particular particle species by making the trivial transformation back to the $(E, F)$ representation. Note that in defining the separatrixes in the $(\theta, \phi)$ space, we have in effect defined the partition of the $(\sigma, \lambda)$ parameter space into regions which do or do not allow trapping. However, all values of $\sigma$ and $\lambda$ do not correspond to real trajectories. It can be shown that for a given $\lambda$, the range of values of $\sigma$ for which can be found real solutions to Eq. (4.2) is $\sigma_{1} \leq \sigma \leq \sigma_{2}$. This condition determines the limits of integration of $F$ in Eqs. (2.20)-(2.22). [The limits of $E$ are obviously $(0, \infty)$ as the integrals are written.] With the thought of making the foregoing study a little more transparent, we show in Figs. 2-4, some of the main features of the particle trajectories in the $(\theta, \phi)$ plane for various values of $\sigma$ and $\lambda$. In these figures the various trapping regions are marked, as well as the separatrixes.

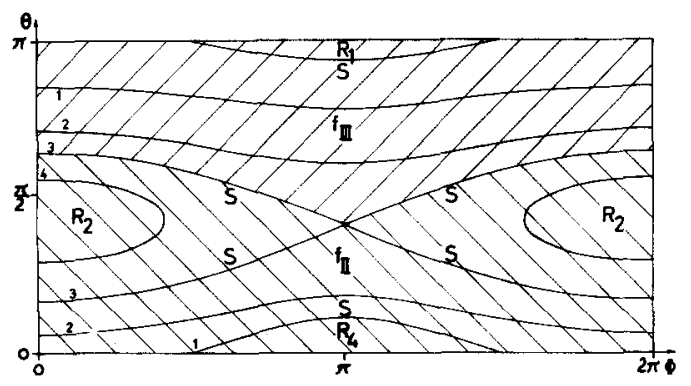

Fra. 3. Sketch of typical phase plane trajectories for $\lambda>\lambda_{c}$. The particle density on any of these trajectories for a given species can be described by two independent distribution functions $f_{\mathrm{II}}$ and $f_{\mathrm{III}}$.

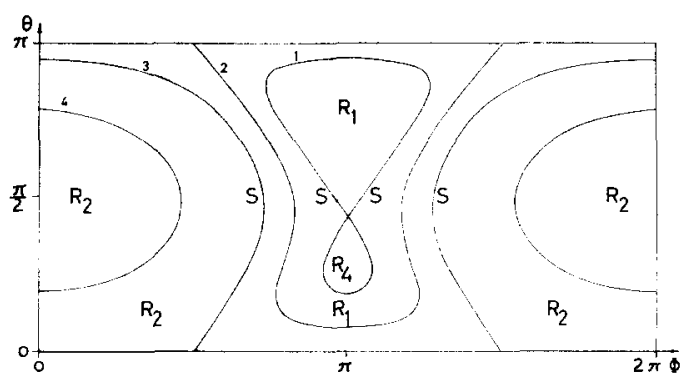

FIG. 4. Typical phase plane trajectories for $\lambda_{d}>\lambda>\lambda_{0}$ and $r$ large. For these values of the parameters; the trapping region $R_{1}$ is no longer simply connected.

Figure 4 is included primarily to show the form of the trajectories for large values of $r$.

Figure 2 shows a typical display of the particle trajectories in the phase plane for those cases where $\lambda<\lambda_{c}$. Here the individual particle species may be described by a single distribution function $f_{\mathrm{I}}$.

Figure 3 is typical of those cases where $\lambda>\lambda_{0}$. Here the individual particle species are described by two independent distribution functions $f_{I I}$ and $f_{\text {III }}$. The domains of these functions are indicated in the figure by cross-hatching. (The definition of the distribution functions is given in the following subsection.)

Figure 4 represents those cases where $\lambda_{d}>\lambda>\lambda_{c}$ and the ratio $r$ is large. Here the point of interest is that the region $R_{1}$ is no longer simply connected and each of the two functions $f_{\mathrm{II}}$ and $f_{\mathrm{III}}$ describe a portion of this region.

In each of Figs. 2-4, the separatrixes are denoted by the letter $\mathrm{S}$, and the trajectories are numbered in increasing order, 1, 2, 3, etc., corresponding to increasing values of the parameter $\sigma$.

\section{B. The Distribution Functions}

In Eqs. (2.20)-(2.23), we have allowed for the fact that the particle distribution functions $f^{(i)}$ may vary over the branches of $\dot{\phi}$ by introducing the subfunctions $f_{s}^{(i)}$ and taking the integrals separately for each branch of $\dot{\phi}$. However, there is a constraint upon $^{9} f$ which will connect some of these subfunctions and which arises because we require the $f$ to be time independent in the rest frame. This constraint is just that the $f$ must be constant along a trajectory. The validity of this condition is made clear by considering that the physical motion of the particles described by a given $f$ and initially located upon a given trajectory will cause them to circulate around the trajectory as time

${ }^{9}$ In what follows, the superscript $(i)$ attached to $f^{(i)}$ is understood. 
progresses, and consequently, the initial values of $f$ along the trajectory must also "circulate." Thus, the value of $f$ on any particular section of the trajectory can remain constant in time only if the initial values of $f$ on all portions of the trajectory are equal.

Since the distribution functions will be constant over a trajectory, we need only apply the subscript $s$ with regard to these functions in order to differentiate between trajectories rather than between branches of $\dot{\phi}$. However, note that this simplification can be made to apply also to the integrals in $\phi$ defined in Eqs. (2.23). From Eqs. (2.11) and (2.12) and the last of Eqs. (2.10), it is evident that the branches of $\phi$ are defined by the branches of $v_{z}(E, F, \phi)$, and clearly, each trajectory will be made up of an integral number of branches of $\dot{\phi}$. But $f^{(i)}$ is constant on any trajectory, so we may combine those integrals in $\phi$ which are to be taken over the branches of $\dot{\phi}$ into a single integral which is to be taken over all the branches of $\dot{\phi}$ which exist on the given trajectory. In this case, then, the subscript $s$ attached to these integrals need also only differentiate between trajectories rather than branches, and henceforth, without loss of generality, we consider that the subscript $s$ as used in Eqs. (2.20)-(2.23) applies to quantities defined over different trajectories.

As mentioned at the start of Sec. IVA, there are at most two trajectories for given values of $\lambda$ and $\sigma$, and thus, it is necessary to specify at most two independent distribution functions $f_{s}$ for any $\lambda$ and $\sigma$. It is convenient to define these independent distribution functions in the following way as functions of $\lambda$ and $\sigma$ :

$f_{\mathrm{I}}$ : Define $f_{\mathrm{I}}$ to be the single distribution function in the phase space region $0 \leq \lambda \leq \lambda_{\mathrm{c}}, \sigma_{2} \geq \sigma \geq \sigma_{1}$. In this range there exists only one trajectory for each $\lambda$ and $\sigma$.

$f_{\text {II }}$ and $f_{\text {III }}$ : In the interval $\lambda_{c} \leq \lambda \leq \infty$, define $f_{\text {II }}$ to be a distribution function for $\sigma_{2} \geq \sigma \geq \sigma_{4}$ and $f_{\text {III }}$ to be an independent distribution function for $\sigma_{3} \geq \sigma>\sigma_{1}$. In the interval of common definition $\sigma_{3} \geq \sigma \geq \sigma_{4}$, let $f_{\mathrm{II}}$ describe the simply connected phase space area which includes the trapping region $R_{4}$. (For $\lambda_{\mathrm{c}} \leq \lambda \leq \lambda_{\mathrm{d}}$, $f_{\mathrm{Ir}}$ will describe only the region $R_{4}$ plus separatrix, in the interval of common definition; but for $\lambda>\lambda_{d}, f_{I I}$ will describe in addition to $R_{4}$ a region of nontrapped particle motion. See Fig. 3.)

In addition, we define each of these three distribution functions to be identically equal to zero for values of $\lambda$ and $\sigma$ outside of the particular ranges listed above.
The above three distribution functions suffice to describe the system; however, in general they will not be completely independent. In most cases of physical interest, we would expect that the over-all distribution of particles would be continuous in $\lambda$ and $\sigma$ for physically meaningful values of $\sigma$. In addition to this requirement, it must also be demanded physically that the population of particles at the unstable singular point $\left(\theta_{3}, \phi_{3}\right)$ be zero. Thus, we arrive at the following set of boundary conditions on the distribution functions:

$$
\begin{aligned}
& f_{\mathrm{I}}\left(\lambda_{o}, \sigma\right)=\begin{array}{ll}
f_{\mathrm{II}}\left(\lambda_{c}, \sigma\right), & \sigma_{2} \geq \sigma \geq \sigma_{4}, \\
f_{\mathrm{III}}\left(\lambda_{c}, \sigma\right), & \sigma_{4} \geq \sigma \geq \sigma_{1},
\end{array} \\
& f_{I I}\left(\lambda, \sigma_{3}\right)=f_{I I I}\left(\lambda, \sigma_{3}\right)=0, \lambda_{c} \leq \lambda \leq \infty .
\end{aligned}
$$

Equation (4.9) is just the statement that the population of the unstable singular point is zero and is interesting in that it implies that if there are any cyclotron-trapped particles, the distribution function cannot be written as the product of two independent distributions in $\lambda$ and $\sigma$, i.e., $f(\lambda, \sigma)=g_{1}(\lambda) g_{2}(\sigma)$. In order to see this let us suppose that the distribution function can be written in product form as above. In this case ${ }^{10} g_{2}(\sigma)$ must satisfy Eq. (4.9), i.e.,

$$
g_{2}\left(\sigma_{3}\right)=0, \quad \lambda_{\mathrm{c}} \leq \lambda \leq \infty .
$$

But this boundary condition can be satisfied only if $g_{2}$ is identically zero over the entire range of values of $\sigma$ that $\sigma_{3}$ may take on as $\lambda$ varies between $\left(\lambda_{e}, \infty\right)$. However, it can be shown from the results of the phase plane analysis that $\sigma_{3}$ is a continuous, monotonically increasing function of $\lambda$, for $\lambda>\lambda_{0}$, which has the limit from the right $\sigma_{\mathrm{c}} \equiv \sigma_{3}\left(\lambda_{\mathrm{c}}\right)$ and which varies as $\lambda^{2}$ for large $\lambda$. Thus the range of $\sigma_{3}$ is $\left(\sigma_{c}, \infty\right)$ as $\lambda$ varies between $\left(\lambda_{0}, \infty\right)$ and $g_{2}$ must satisfy

$$
g_{2}(\sigma)=0, \quad \sigma_{\mathrm{c}} \leq \sigma \leq \infty .
$$

But the phase plane analysis also shows that the cyclotron trapped particles will possess values of $\sigma$ only in the range $\sigma_{2} \geq \sigma>\sigma_{3}$, and thus, any cyclotron trapped particle will possess a value of $\sigma$ which is greater than $\sigma_{\mathrm{a}}$. Consequently, the presence of any cyclotron-trapped particles means that $g_{2}(\sigma) \neq 0$ for some $\sigma>\sigma_{\mathrm{e}}$, and this violates Eq. (4.11). This contradiction implies that the distribution function cannot be written as the product of two independent distributions in $\sigma$ and $\lambda$ if there are any cyclotron trapped particles in the system. As a corollary of this result, it can be stated that in general the only

${ }_{10}$ The same type of argument as used here will apply if a part, or all, of the burden of satisfying Eq. (4.9) is shifted upon the function $g_{1}$. 
isotropic distribution functions, i.e., $f(\sigma, \lambda)=f(\lambda)=$ $f\left(v^{2}\right)$, which satisfy the boundary conditions are those which vanish for $\lambda \geq \lambda_{c}$.

\section{Integrals over $\phi$}

It remains to discuss the form of the integrals over $\phi$ in Eqs. (2.23). These integrals represent in essence a time weighting of the various integrands, and in view of the fact that each of the distribution functions will be constant along any trajectory which lies within their domain, this time weighting may always be taken over an entire trajectory, as discussed previously. From Eqs. (2.11) and (2.22), it can be seen that it is possible to express the quantity $v_{\perp} \cos \phi$ in terms of a second degree polynomial in $v_{z}$, and consequently, each set of $T_{s}^{(i)}$, $H_{s}^{(i)}, G_{s}^{(i)}$ for fixed $s$ and $i$ will involve only linear combinations of the time weighted values of $\left(v_{z}\right)^{p}$ along a given trajectory, where $p=0,1,2$.

It is convenient to carry out the time weightings in terms of a normalized variable rather than $\phi$. Let us introduce the variable $Y=v_{z} V_{0}^{-1}$. Over a trajectory the differential relation holds

$$
d \tau=d \phi / \phi=d v_{z} / \dot{v}_{z}=d Y / \dot{Y},
$$

where $d \tau$ is a differential time element along the trajectory and, by convention, is to be considered a positive quantity. Utilizing the first of Eqs. (2.10) in addition to Eqs. (2.11) and (2.12), it is possible to find $\dot{Y}$ in terms of $Y$ alone, and $d \tau$ may be expressed

$$
\begin{aligned}
d \tau & =2 \pi_{i}\left\{4 r^{2}\left[\lambda^{2}-Y^{2}\right]-\left[\gamma+(1-Y)^{2}\right]^{2}\right\}^{-\frac{1}{2}} d Y \\
& \equiv 2 \pi_{i}[P(Y)]^{-\frac{1}{2}}
\end{aligned}
$$

where

$$
\gamma=\sigma-\lambda^{2}-1, \quad \pi_{i}=\left(\eta_{i} B_{0}\right)^{-1} .
$$

It is wished to evaluate integrals of the sort

$I_{p}=\int_{\mathrm{s}}\left(v_{z}\right)^{p} \frac{d \phi}{J_{\mathrm{s}}}=\frac{k V_{\mathrm{o}} p}{4} \oint Y^{p} d \tau=\frac{k V_{\mathrm{c}} p}{2} \oint Y^{p} d \tau$,

where the full, or half, circle indicate that the integration is to be taken along the full, or half, trajectory. The last equality follows because according to Eq. (2.12) every trajectory will be symmetric in $\phi$ about $\phi=0$ and $\pi$. The end points of this half-trajectory will be appropriate values of $Y$ at $\phi=0$ and/or $\pi$. It can be shown from Eq. (4.12) and Eqs. (2.10) that $d \tau / d Y$ is a single-valued function of $Y$ on the half-trajectory (excluding end points) and that the end points of the trajectory are adjacent real zeros of $P(Y)$. Consequently, $I_{p}$ may be written

$$
I_{p}=\left|V_{c}\right|^{-1} V_{c}^{v} \int_{m}^{n} Y^{p}[P(Y)]^{-\frac{1}{2}} d Y,
$$

where

$$
P(n)=P(m)=0, \quad n>m .
$$

(The absolute value of $V_{0}$ arises because $d \tau$ is taken in a positive sense.)

The polynomial $P(Y)$ is a quartic in $Y$, and the integrals $I_{p}$ may, consequently, be expressed in terms of the complete elliptic functions of the first and second kinds and the Heuman lambda function. ${ }^{11}$ These functions are well known and have been extensively tabulated. We do not give here the exact form of $I_{p}$ in terms of these complete elliptic functions since this representation may be obtained from standard handbooks. ${ }^{12}$ We merely indicate below the proper limits of integration $(m, n)$ for the $I_{p}$ in the various domains of the particle distribution functions $f_{\mathrm{s}}, S=$ I, II, III:

Domain I: $P(Y)$ possesses only two real roots for $0 \leq \lambda<\lambda_{\mathrm{c}}$. Let these be $a \geq b$; then $m, n=b, a$. For $\lambda=\lambda_{c}, a$ is a triple root of $P$ at $\sigma=\sigma_{3}$, but the limits of integration remain the same.

Domain II: Here $\lambda \geq \lambda_{0}$. For $\sigma_{2} \geq \sigma>\sigma_{3}$, $P$ has only two real roots $b \geq c$, and $m, n=c, b$. For $\sigma_{4} \leq \sigma \leq \sigma_{3}, P$ has four real roots $a \geq b \geq c>d$, and $m, n=b$, $a$.

Domain III: Here $\lambda>\lambda_{\mathrm{c}}$. For $\sigma_{4} \leq \sigma \leq \sigma_{3}$, $P$ has four roots $a \geq b \geq c>d$, and $m, n=d, c$. For $\sigma_{1} \leq \sigma<\sigma_{4}, P$ has two real roots $c \geq d$ and $m, n=d, c$.

Since the roots of $P(Y)$ depend upon $\lambda$ and $\sigma$, the $I_{p}$ will be functions of $\lambda$ and $\sigma$, also. However, the dependence of $I_{p}$ upon the explicit values of charge or mass of any particular particle species will enter only through the multiplying factor which precedes the integral in $Y$ in Eq. (4.13).

\section{Final Integral Equations}

With the results given thus far in this section, it would now be possible to express Eqs. (2.20)-(2.23) fully in terms of the quantities $E$ and $F$ and $\phi$. However, it is convenient to remain in the $(\lambda, \sigma, Y)$ representation since in this representation the original system of integral equations may be written in a more compact form. For the final equations we introduce the following functions:

$$
\begin{gathered}
f_{s}^{(t)}=\sum_{i} \epsilon_{i}\left|V_{\mathrm{o}}\right|^{3} f_{s}^{(i)}(\lambda, \sigma), \\
M_{\mathrm{a}}=\sum_{i}\left|\epsilon_{i}\right| V_{\mathrm{o}}^{4} f_{s}^{(i)}(\lambda, \sigma),
\end{gathered}
$$

${ }^{11}$ C. Heuman, J. Math. Phys. 20, 127 (1941).

${ }^{12}$ See, for example, P. F. Byrd and M. D. Friedman, Die Grundlehren der Mathematischen Wissenschaften, of Handbook of Elliptic Integrals for Engineers and Physicists (SpringerVerlag, Berlin, 1954), Vol. 67. 


$$
\begin{aligned}
& T(m, n)=\int_{m}^{n}[P(Y)]^{-\frac{1}{3}} d Y, \\
& H(m, n)=\int_{m}^{n} Y[P(Y)]^{-\frac{1}{2}} d Y, \\
& G(m, n)=\int_{m}^{n}\left[\gamma+(1-Y)^{2}\right][P(Y)]^{-\frac{1}{2}} d Y,
\end{aligned}
$$

where $\epsilon_{i}$ is defined by $q_{i}=\epsilon_{i} e, e$ is the magnitude of the electronic charge, and $m$ and $n$ are adjacent roots of $P(Y)$ as described in Sec. IVC.

In terms of the above quantities, Eq. (2.20) takes the final and explicit form

$$
\begin{aligned}
K= & \int_{0}^{\lambda_{0}} \lambda d \lambda \int_{\sigma_{1}}^{\sigma_{3}} d \sigma M_{\mathrm{I}} G(b, a) \\
& +\int_{\lambda_{\varepsilon}}^{\infty} \lambda d \lambda\left\{\int_{\sigma_{s}}^{\sigma_{2}} d \sigma M_{\mathrm{II}} G(c, b)\right. \\
& +\int_{\sigma_{4}}^{\sigma_{3}} d \sigma\left[M_{\mathrm{II}} G(b, a)+M_{\mathrm{III}} G(d, c)\right] \\
& \left.+\int_{\sigma_{2}}^{\sigma_{*}} d \sigma M_{\mathrm{III}} G(d, c)\right\},
\end{aligned}
$$

where

$$
K=k B_{w}^{2} / e \mu_{0} B_{0} .
$$

For the sake of brevity we do not write Eqs. (2.21) and (2.22) out in full; however, Eq. (2.21) may be obtained in explicit form from Eq. (4.14) by setting $K$ equal to zero and replacing the quantity $G(m, n)$, wherever it occurs, by the quantity $H(m, n)$. Equation (2.22) may be obtained in explicit form from Eq. (4.14) by setting $K$ equal to zero and replacing the quantity $M_{s} G(m, n)$, wherever it occurs, by the quantity $f_{s}^{(t)} T(m, n)$.

In symbolic notation Eqs. (2.20)-(2.22) now have the final form:

$$
\begin{aligned}
K & =\int \lambda d \lambda \int d \sigma M(\sigma, \lambda) G(m, n), \\
0 & =\int \lambda d \lambda \int d \sigma M(\sigma, \lambda) H(m, n), \\
0 & =\int \lambda d \lambda \int d \sigma f^{(t)}(\sigma, \lambda) T(m, n),
\end{aligned}
$$

with_the boundary conditions

$$
\begin{aligned}
M\left(\lambda, \sigma_{3}\right) & =0, \quad \lambda \geq \lambda_{0}, \\
f^{(t)}\left(\lambda, \sigma_{3}\right) & =0 .
\end{aligned}
$$

\section{DISCUSSION}

At the present time a program of numerical and theoretical investigation of Eqs. (4.15) is being carried out at the Ionosphere Laboratory of the Technical University of Denmark with the aim of obtaining some general ideas of the effects that the cyclotron-trapped particles have upon nonlinear whistler mode waves. This study has applications to certain theories ${ }^{13,14}$ of very low frequency emissions $^{15}$ in which it proposed that the emissions are generated through a plasma instability involving cyclotron trapped particles. A question arises in these theories as to the number of particles that a given whistler mode wave can trap, and while our study cannot answer this question directly, it should serve to give a better estimate of this number than has been previously available from the nonlinear cold plasma theory. However, our study has not been completed to date, and we have no final numerical results to present in this paper. Our main thought in presenting here the analytical development of the dispersion relations is that these relations might prove of interest to other workers.

Two questions which certainly warrant further investigation are that dealing with the general method of inversion of Eqs. (4.15) in order to find the distribution functions explicitly and that dealing with the stability of the solutions to Eqs. (4.15). The question of stability seems the more complex one since the stability of the nonlinear longitudinal plasma oscillations has not been conclusively shown, ${ }^{16}$ although these waves represent a simpler system than ours.

In closing, it should be remarked that the domains of the distribution functions as given in Sec. IV were chosen solely for convenience in studying the cyclotron-trapped particles and will not necessarily be appropriate if it is wished to study some other trapping region. Nevertheless, a boundary condition similar to that of Eq. (4.9) must apply no matter how the domains of the distribution functions are chosen, and it will remain the case that the presence of cyclotron trapped particles imposes important constraints as to the form of the distribution functions which will satisfy Eqs. (4.15).

\section{ACKNOWLEDGMENTS}

The author is grateful to the Ionosphere Laboratory of the Technical University of Denmark and especially to Professor J. Lindhard and Dr. E. Ungstrup, whose support made this study possible. Special thanks are due to Torben Stockflet Jørgensen, Peter Stauning, and Jorgen Perrson who aided in the preparation of this manuscript.

${ }^{13}$ N. M. Brice, J. Geophys. Research 68, 4626 (1963).

${ }^{14} \mathrm{~S}$. F. Hansen, J. Geophys. Research 68, 5925 (1963).

15 R. A. Helliwell, J. Geophys. Research 68, 5387 (1963).

${ }^{16}$ D. C. Montgomery and D. A. Tidman, Plasma Kinetic Theory (McGraw-Hill Book Company, Inc., New York, 1964), Chap. 11. 